\title{
ENTRE RÉTRO ET FUTURISME. LE ZEPPELIN DANS LA CULTURE VISUELLE CONTEMPORAINE
}

\author{
Sébastien Fevry ${ }^{1}$
}

\begin{abstract}
Cet article vise à étudier la culture visuelle qui entoure le zeppelin en portant attention non pas tellement à l'évolution de l'objet technologique lui-même, mais à la trajectoire de ses images, particulièrement dans la culture contemporaine. Le propos sera d'établir la biographie de l'image-zeppelin en suivant l'évolution d'un imaginaire technologique, mais également politique, en lien avec la question des utopies.
\end{abstract}

Cet article a pour objectif d'étudier la culture visuelle qui entoure le zeppelin en retraçant ce que Pascal Ory dans L'histoire culturelle (2004) a nommé une imagerie, c'est-à-dire les actualisations successives d'un imaginaire donné à travers le temps ${ }^{2}$. Il s'agit de porter attention non pas tellement à l'évolution de l'objet technologique lui-même, mais à la trajectoire de ses

1 Sébastien Fevry est professeur à l’Université catholique de Louvain.

2 Nous nous rattachons ici à la définition large de l'imaginaire social explicitée par Patrice Leblanc (1994) pour lequel l'imaginaire social peut être considéré comme ayant trait à des systèmes de représentations partagés collectivement comme les mythes, la religion, la mémoire ou, dans notre cas, l'ensemble des représentations relatives à une technologie donnée. Pour Ory, la différence entre imaginaire et imagerie tient surtout à la perspective déployée par le chercheur : les imaginaires s'apparentent à des systèmes qui sont « des constructions ayant une cohérence contemporaine, alors que les imageries n'existeront qu'au travers du regard $a$ posteriori de l'historien » (Ory, 2004, p. 91). 
images, particulièrement dans la culture contemporaine. Même si nous emploierons parfois le terme 'dirigeable' dans la suite de l'article, nous ciblons bien ici le 'zeppelin'3 , à savoir l'aéronef à structure métallique rigide mis au point par le comte Ferdinand von Zeppelin au début du $20^{\mathrm{e}}$ siècle à Friedrichshafen sur les bords du lac de Constance et dont différents modèles allaient se succéder jusqu'à la fin des années $30^{4}$.

L'accent mis sur la culture visuelle du zeppelin se justifie par deux raisons. Tout d'abord, le zeppelin constitue un objet hors norme par ses proportions (246 mètres de long pour le plus grand d'entre eux, le LZ 129 Hindenburg), c'est-à-dire un objet trop grand et trop vaste pour être manipulé. Cela revient à souligner que le lien privilégié entretenu avec le zeppelin est d'abord un rapport d'ordre visuel. À la différence de moyens de transport comme le paquebot ou le train, il y eut relativement peu de personnes qui expérimentèrent un vol à bord d'un dirigeable. Entre 1900 et 1939, seulement 119 engins furent construits (de Syon, 2002, p. 3), ce qui montre bien le caractère limité et exclusif de cette expérience qui était surtout réservée à des voyageurs fortunés, si on excepte l'utilisation de la machine à des fins militaires lors de la Première Guerre mondiale ou à des buts exploratoires lors du survol du pôle Nord au début des années 30 .

Ensuite, deuxième raison, le zeppelin n'a pas connu de réelles postérités technologiques, alors qu'il était pressenti au début du $20^{\text {e }}$ siècle comme étant la voie royale pour conquérir les airs. C'est l'avion qui a rempli ce rôle et le zeppelin n'a pas engendré de véritables descendances, mis à part certains dirigeables à vocation publicitaire ou touristique. Le zeppelin, en tant qu'objet, ne circule plus dans notre environnement contemporain. Son existence nous est rappelée par le biais d'images, de magazines ou de films, lesquels réactivent la présence de cette machine disparue avant la Seconde Guerre mondiale, en 1937, lors de l'accident

3 En allemand, le terme 'zeppelin' désigne par extension n'importe quel type de ballon dirigeable.

4 Cette focalisation sur le zeppelin écarte de notre corpus de départ l'imaginaire du dirigeable qui s'est déployé avant le zeppelin et que l'on retrouve par exemple dans les illustrations d'Albert Robida (1848-1926) qui ont également eu un impact important sur le rétro-futurisme. 
spectaculaire de Lakehurst dans le New Jersey, quand le zeppelin Hindenburg, parti de Francfort, s'enflamma brusquement devant les caméras d'actualité, causant la mort de 35 personnes et marquant la fin de l'aventure des dirigeables en tant que moyen de locomotion ${ }^{5}$.

Ces remarques préliminaires justifient que l'on situe l'étude du zeppelin au sein d'une culture visuelle prenant en compte à la fois le caractère extraordinaire de l'objet et le fait qu'il n'est accessible qu'à travers des représentations. Curieusement, les rares auteurs comme Guillaume de Syon (2002) ou C. Michael Hiam (2014) qui se sont intéressés à la culture du zeppelin se sont surtout limités à la période d'avant 1940, sans prendre en compte les avatars contemporains du dirigeable qui apparaissent pourtant fréquemment dans les univers rétro-futuristes véhiculés par l'esthétique steampunk, les bandes dessinées de Schuiten et Peeters ou certaines installations d'art contemporain comme celles d' Héctor Zamora ou de Panamarenko.

Dans le cadre de cet article, notre propos sera donc d'établir la biographie de l'image-zeppelin en suivant l'évolution d'un imaginaire technologique, mais également politique, en lien avec la question des utopies. Cette démarche peut être mise en parallèle avec des approches issues des Visual Studies, lesquelles postulent qu'une image peut voyager à travers le temps et s'incarner à chaque étape temporelle au sein des médiums particuliers, euxmêmes situés dans des contextes socioculturels déterminés. Dans Pour une anthropologie des images (2004), Hans Belting souligne que « les images ressemblent à des nomades qui auraient changé de mode de vie au gré des cultures traversées dans leur histoire, en utilisant les médiums des diverses époques comme des étapes dans le temps » (pp. 46-47).

Pas plus qu'une autre image, l'image du zeppelin ne voyage seule. Elle emmène avec elle les affects et les discours qui lui

5 Pour beaucoup de commentateurs, la disparition des dirigeables eut comme principale cause la présence des caméras qui diffusèrent les images de l'accident à l'échelle planétaire, ruinant du même coup la réputation de ce moyen de transport. Cette hypothèse explicative est nuancée par Guillaume de Syon dans son ouvrage (2002, pp. 195-203). 
étaient au départ associés. Or, notre hypothèse est d'avancer que le voyage d'une image implique aussi la reconfiguration de l'imaginaire et des émotions qui lui étaient de prime abord attachés. En ce qui concerne le zeppelin, nous chercherons à montrer comment le sentiment du sublime ${ }^{6}$ depuis toujours relié à son image se reconfigure dans des représentations visuelles qui peuvent aussi bien faire le deuil d'utopies passées qu'en projeter de nouvelles. Nous nous intéresserons donc ici à la question du maintien, mais aussi du renouvellement d'une imagerie donnée.

D'un point de vue méthodologique, suivre la trajectoire d'une image implique la sélection de certains moments visuels qui sont certes significatifs en soi, mais qui valent surtout par les similitudes et les différences qu'ils permettent de tisser entre eux. C'est ce que Georges Didi-Huberman appelle une connaissance par le montage ${ }^{7}$. Cette approche n'autorise « ni que l'on mette chaque image en quarantaine, ni que l'on puisse tout mettre sur le même plan »'(2003, p. 152). Dans notre perspective, il s'agit moins de dresser un panorama complet des différentes occurrences du zeppelin dans la culture contemporaine que de composer une trame visuelle permettant de faire surgir des différences signifiantes et de donner lieu à un geste critique qui puisse reconstituer a posteriori les modifications de trajectoire d'une image donnée.

\section{Port d'attache : constitution d'un imaginaire}

Avant de nous aventurer vers la culture contemporaine, il importe de bien saisir ce qui se noue autour de l'image du zeppelin. Comme point de départ, examinons un extrait de l'œuvre de Stefan Zweig Le Monde d'hier. Souvenirs d'un Européen (1993). L'écrivain autrichien écrit cet ouvrage testamentaire alors qu'il s'est exilé au Brésil, peu avant son suicide en 1942. Il y retrace

6 Pour une première approche du sublime et une mise en contexte de ce régime esthétique, voir notamment Edouard Aujaleu (1997).

7 Pour Didi-Huberman, « il faudrait concevoir, par-delà le modèle du procédé graphique ou cinématographique en tant que tel, une notion du montage qui serait au champ des images ce que la différenciation signifiante fut au champ du langage dans la conception post-saussurienne » (2003, p. 152). 
l'évolution de l'Europe depuis la période d'insouciance précédant la Première Guerre mondiale jusqu'à la montée des totalitarismes et l'apparition de la barbarie nazie.

Dans le chapitre intitulé "Les rayons et les ombres sur l'Europe », Zweig consacre un court passage au zeppelin :

Le jour où le Zeppelin prit son vol pour son premier voyage, j'étais par hasard de passage à Strasbourg, me rendant en Belgique ; il tourna autour de la cathédrale aux acclamations enthousiastes de la foule, comme s'il voulait, lui qui flottait dans les airs, s'incliner devant l'œuvre millénaire. Le soir, en Belgique, chez Verhaeren, arriva la nouvelle que le dirigeable s'était écrasé à Echterdingen. Verhaeren avait les larmes aux yeux et était terriblement agité. Loin d'être, en tant que Belge, indifférent à la catastrophe qui frappait l'Allemagne, en tant qu'Européen, en tant qu'homme de notre temps, il éprouvait aussi vivement la victoire commune sur les éléments que la commune épreuve. (Zweig, 1993, p. 234)

Le voyage du zeppelin relaté par Zweig peut être daté avec précision. Il s'agit du premier long voyage du dirigeable en août 1908 à travers l'Europe qui se termina effectivement par le crash de la machine à Echterdingen, un village près de Stuttgart. Deux images permettent d'illustrer le témoignage de Zweig : tout d'abord, une carte postale du zeppelin au-dessus de Strasbourg (voir figure 1). On peut d'ailleurs se demander si l'écrivain était présent ce jour-là à Strasbourg ou si c'est plus tard, devant une carte postale similaire, qu'il imagina la scène où le zeppelin s'incline devant la cathédrale. Ensuite, deuxième image, une photographie attribuée à Franz Stoedtner (voir figure 2), reprise dans différents journaux de l'époque, montrant les débris du zeppelin après la catastrophe. 


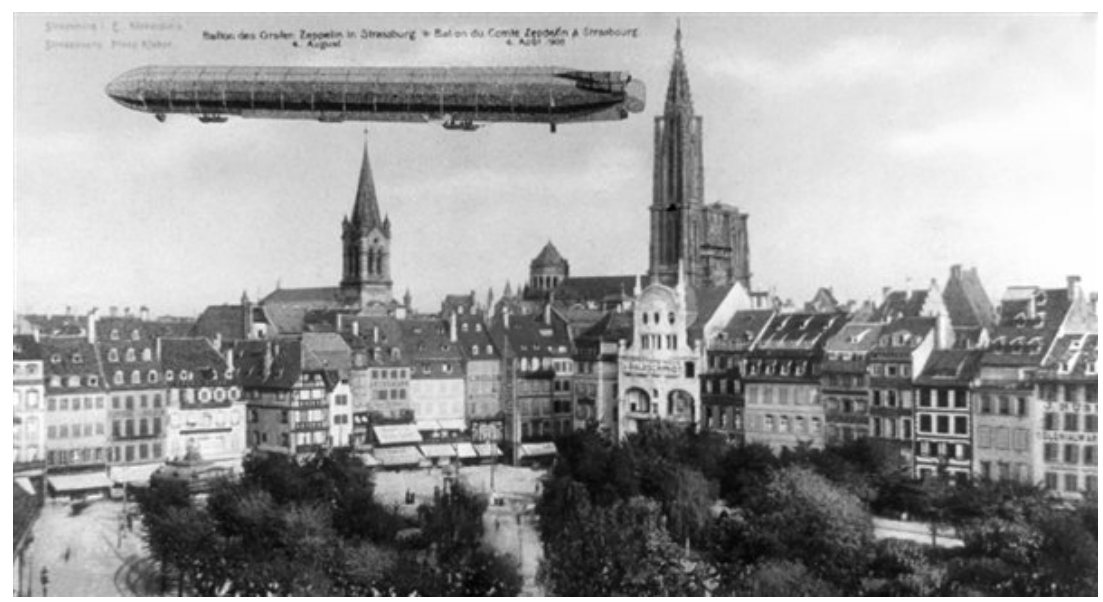

Figure 1. Le zeppelin au-dessus de Strasbourg

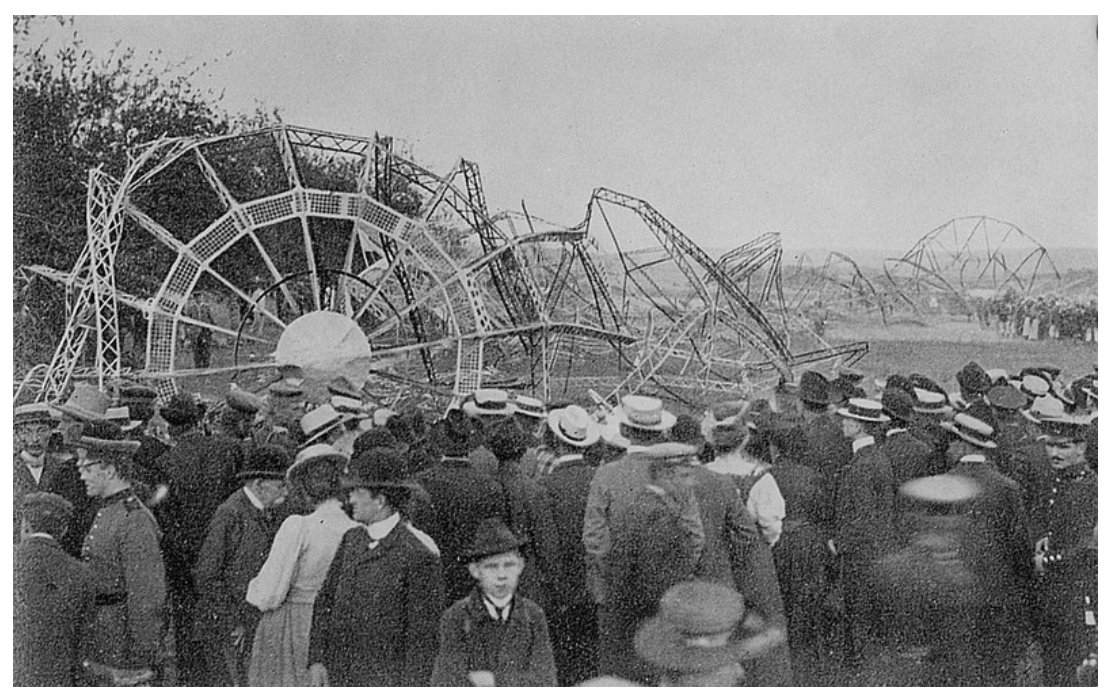

Figure 2. L'accident d'Echterdingen

En termes de culture visuelle, ces images indiquent déjà les deux grandes séries visuelles qui se trouvent mobilisées autour de la représentation du dirigeable. D'une part, une famille d'images que l'on peut associer au paradigme du voyage comprenant des cartes postales, des affiches pour des destinations lointaines, ou 
encore des timbres à l'effigie du zeppelin ${ }^{8}$. D'autre part, une série visuelle que l'on peut associer au paradigme de la catastrophe, laquelle mobilise des photographies de presse ou des films d'actualité comme celui qui sera tourné lors du crash de Lakehurst en 1937. Au-delà des accidents, ce paradigme peut être élargi aux images comportant une dimension apocalyptique et présentant le zeppelin comme un danger potentiel, ce qui implique d'intégrer des actualités de guerre ou des caricatures figurant le zeppelin comme une menace pour les pays alliés. Les deux séries d'images peuvent évidemment se recouper, mais il importe de distinguer d'emblée ces deux orientations figuratives.

L'extrait de Zweig aide aussi à mieux délimiter l'imaginaire du zeppelin en regard du tissu culturel de l'époque : la représentation du dirigeable se trouve à la fois liée à un certain type d'acteur, à un certain type d'émotion et à un discours comportant une nette dimension utopique.

Le partenaire privilégié du zeppelin, son interlocuteur principal, c'est la masse. Stefan Zweig écrit que le passage du zeppelin au-dessus de Strasbourg suscita l'enthousiasme de la foule, ce qui est conforté par d'autres descriptions historiques qui pointent le fait que l'arrivée d'un dirigeable suscitait fréquemment le rassemblement d'un grand nombre d'individus'. Le dirigeable, moyen de transport réservé à une élite, peut se concevoir comme un spectacle de masse, contemplé depuis le sol par une foule compacte, un agrégat de personnes faisant corps devant l'enthousiasme soulevé par le passage de la machine. Si cette euphorie collective a toujours été soigneusement entretenue, voire amplifiée, par les différents régimes politiques qui se succèdent en Allemagne, elle se trouve particulièrement accentuée et mise en scène sous le Troisième Reich, lorsque le zeppelin sert

8 Pour une iconographie de la culture du zeppelin incluant notamment les timbresposte, on se rapportera à l'ouvrage illustré de Brigitte Kazenwadel-Drews (2006).

9 Sur Internet, le site du Rebstockpark de Francfort rappelle que, lors de l'atterrissage du premier zeppelin à Francfort en 1909, les clameurs de la foule étaient si intenses que le maire de la ville fut incapable de faire entendre son discours de bienvenue au comte von Zeppelin qui avait accompagné l'appareil.

À ce sujet, voir www.rebstockpark-ffm.de/rebstock.htm (consulté le 10 mai 2017). 
d'instrument de propagande au régime nazi, notamment lors des Jeux olympiques de 1936 (de Syon, 2002, pp. 172-186).

La foule est reliée au zeppelin par un type particulier d'émotion que Zweig appelle enthousiasme, mais qu'un auteur comme Guillaume de Syon préfère appeler le 'sublime technologique' ${ }^{\prime 10}$. Dans son acception traditionnelle, chez des auteurs comme Burke ou Kant, le sublime est rattaché à des spectacles de la nature comme les tempêtes ou les tremblements de terre qui, par leur caractère extraordinaire, provoquent à la fois un sentiment de fascination et de crainte. Pour de Syon, c'est un même type de sentiment que l'on peut associer au zeppelin, sauf qu'ici ce sentiment n'est pas suscité par un spectacle de la nature, mais par une nouvelle invention technique dont les dimensions et les prouesses semblent excéder les limites de l'imagination. Les projets technologiques de grande taille comme le zeppelin dépassent en effet l'entendement : ils nous demandent « d'ajuster nos facultés de perception à un objet si immense que cela nous conduit à un état proche du ravissement $\rangle^{11}$ (de Syon, 2002, p. 4).

Toutefois, faire porter le sublime du zeppelin sur la seule prouesse technologique est méconnaître qu'à ce sublime technologique s'adjoint aussi un sublime que nous qualifierons de sublime architectural. Ce dernier est lié au fait que le zeppelin paraît constitué comme un monument du ciel, reprenant à son compte l'héritage des constructions et des édifices les plus prestigieux. À cet égard, cen'est pas un hasard que Zweig choisisse de décrire le salut du zeppelin à la cathédrale de Strasbourg. Il y a là comme un passage de relais d'une monumentalité à une autre, d'une verticalité à une horizontalité. D'un point de vue visuel, de nombreuses images de l'époque jouent sur cette proximité comme s'il s'agissait de communiquer aux zeppelins la majesté des hauts bâtiments urbains, d'abord en Europe (voir figure 3) puis aux États-Unis. Sur de nombreux clichés, le zeppelin est

10 La notion de sublime technologique est d'abord appliquée par David Nye (1994) aux constructions architecturales et aux exploits technologiques qui apparaissent aux États-Unis, depuis le pont de Brooklyn jusqu'au lancement des premières fusées.

11 Notre traduction de l'anglais : « to adjust our powers of perception to an object so huge that it leads to a state of near rapture ». 
photographié autant comme un nouvel élément architectural que comme un moyen de transport, ce qui fait que le sentiment de sublime résulte aussi bien d'une prouesse technique que de l'impression proprement sidérante de contempler un monument en plein ciel.

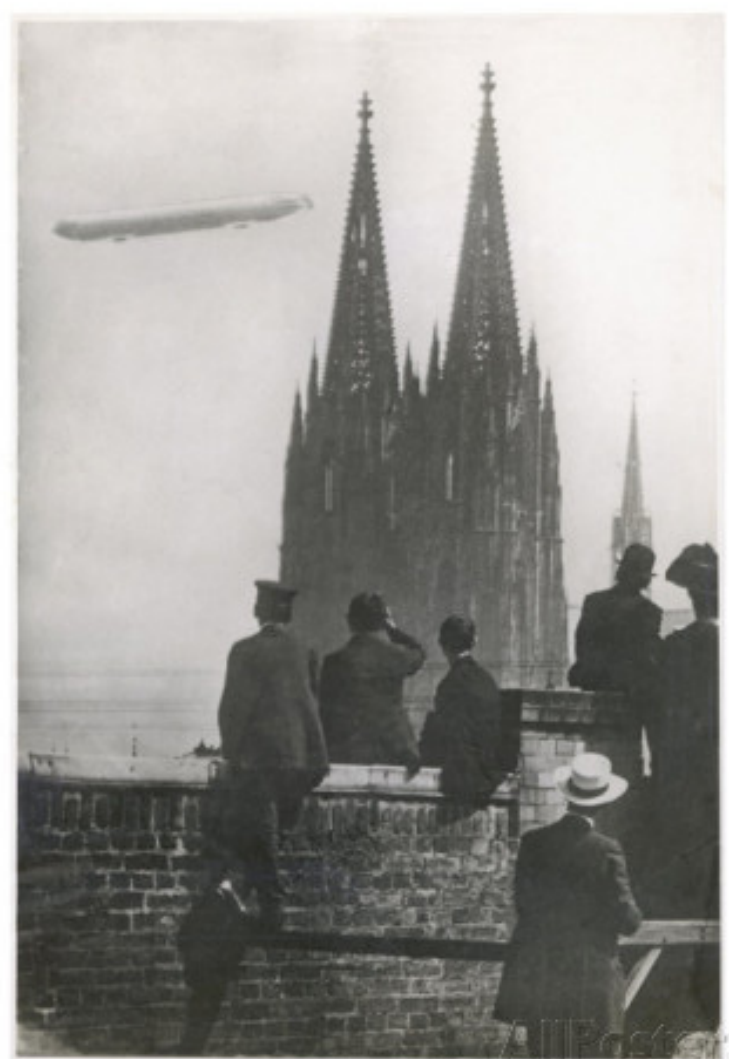

Figure 3. Le zeppelin au-dessus de la cathédrale de Cologne (1909)

Chez Zweig, l'émotion de masse provoquée par le zeppelin soutient plus largement une utopie du progrès et du devenir possible de l'Europe. Dans le même chapitre, Zweig évoque d'autres exploits technologiques comme la traversée de la Manche par Louis Blériot, l'apparition de l'automobile ou 
du chemin de fer électrique : "Grâce à la fierté qu'inspiraient à chaque heure les triomphes sans cesse renouvelés de notre technique, de notre science, pour la première fois, un sentiment de solidarité européenne, une conscience nationale européenne était en devenir » (Zweig, 1993, p. 234).

Cependant, lorsqu'il écrit ces lignes, Zweig est exilé au Brésil à la suite des montées du totalitarisme en Europe et il ne peut ignorer que ce sentiment de solidarité appartient à une histoire passée. L'évocation de l'idéal européen s'effectue depuis un temps et un lieu où celui-ci n'a plus cours. Plutôt qu'une solidarité entre les peuples de l'Europe, Zweig voit surgir son exact envers qui débouchera bientôt sur la Deuxième Guerre mondiale. C'est pourquoi le vol du Zeppelin est si emblématique pour Zweig, puisque la catastrophe d'Echterdingen marque, dans le texte, le passage d'une « victoire commune » à une « commune épreuve ", comme si, à travers cette catastrophe aéronautique, Zweig relisait le destin européen, de son aube au crépuscule, dans un chapitre qui s'intitule précisément « Les rayons et les ombres sur l'Europe ».

Si l'on rapporte le passage de Zweig au contexte de son écriture, on pourrait avancer que se croisent autour du zeppelin les deux faces de l'utopie envisagées par Paul Celan ${ }^{12}:$ l'utopie comme ce qui est de l'ordre du réalisable et l'utopie comme non-lieu ou vision chimérique. Chez Zweig, on perçoit bien l'oscillation entre ces deux faces : le désir utopique d'une société européenne unifiée grâce au progrès technologique est évoqué par un discours rédigé a posteriori qui frappe ce désir d'impossibilité. Ce passage d'une face à l'autre de l'utopie peut être étendu audelà de l'œuvre de Zweig. Que ce soit à l'orée du $20^{\mathrm{e}}$ siècle ou dans les années 20 lorsque le zeppelin entame les premiers vols transatlantiques, le dirigeable est certes porté par l'élan utopique du progrès et d'une société moins repliée sur ses frontières,

12 Pour plus de précision au sujet de la distinction de Paul Celan, on se rapportera à Enzo Traverso (2016, pp. 143-144). Cette distinction recoupe celle effectuée par Cornelius Castoriadis entre projet et utopie : «alors que l'utopie s'apparente à une 'sorte d'étoile polaire' inaccessible et finalement impropre à l'action, le projet (d'autonomie) a non seulement déjà été réalisé au cours de l'histoire [...), mais peut (et doit) encore se réaliser ici et maintenant » (Michel, 2016, p. 29). 
mais parallèlement, cette utopie d'ouverture au monde, bien que fondamentalement eurocentriste, est constamment menacée par son envers, ainsi qu'en attestent les usages militaires du zeppelin pendant la Première Guerre mondiale et sa récupération par la propagande du Troisième Reich dans les années 30 .

À travers cette analyse, on voit combien l'image du zeppelin, répartie entre le paradigme du voyage et celui de la catastrophe, est profondément ambivalente tant sur un plan émotionnel que discursif, puisque le zeppelin peut aussi bien constituer l'aube d'un temps nouveau que représenter un appareil dangereux, porteur des pires catastrophes. Il y a donc là le socle d'une imagerie qu'il nous faut maintenant confronter à la culture visuelle contemporaine pour voir comment se retrouvent redistribués ces éléments constitutifs de l'image-zeppelin que sont la foule, le rapport à l'architecture et la dimension utopique.

\section{Zeppelins et univers rétro-futuristes}

Si l'on met de côté les images du dirigeable apparaissant dans certaines reconstitutions historiques ${ }^{13}$, force est de constater que le zeppelin se trouve aujourd'hui fréquemment associé à des temporalités déviantes ou parallèles que l'on peut regrouper sous l'étiquette générale de rétro-futurisme ${ }^{14}$. Par rétro-futurisme, il ne faut pas seulement entendre le courant spécifique du steampunk ${ }^{15}$, mais plus largement, pour reprendre les termes de Fabien Delrue (2015), « toutes les pratiques culturelles qui télescopent des visions du passé avec des visions du futur ». Dans cette mouvance assez large regroupant aussi bien des films d'animation de

13 On peut penser à Indiana Jones et la Dernière croisade (Spielberg, 1989) ou au long-métrage Un long dimanche de fiançailles (Jeunet, 2004) avec la séquence d'un zeppelin qui s'enflamme dans un hangar lors la Grande Guerre.

14 Une des sources du rétro-futurisme peut être cherchée dans l'imaginaire dystopique qui se met en place à partir de la seconde moitié du $19^{\mathrm{e}}$ siècle, en France notamment. À ce sujet, voir l'ouvrage de Clément Dessy et Valérie Stiénon (2015).

15 Dans Tout le steampunk! (Barillier \& Colson, 2014), les auteurs citent la définition d'un dictionnaire de référence américain qui a le mérite de poser une première délimitation du phénomène. Le steampunk y est entendu comme « science-fiction dealing with 19 th-century societies dominated by historical or imagined steampowered technology » (p. 4). 
Miyazaki que des albums de Schuiten et Peeters ou Andreas ${ }^{16}$, sans compter la série télévisée Fringe (FOX, 2008-2013) ou des productions plus directement affiliées au steampunk comme les bandes dessinées Le Régulateur (Corbeyran, Moreno, 20022014) ou Hauteville House (Duval, Gioux, 2004-), la silhouette du zeppelin s'avère pour beaucoup " incontournable, dans la mesure où elle peut désigner à elle seule le monde rétro-futuriste, telle une estampille » (Barillier \& Colson, 2014, p. 262).

La présence importante du zeppelin dans l'univers rétrofuturiste peut s'expliquer par le fait que le dirigeable pénètre dans la culture contemporaine sous le signe d'une double perte. La perte première est la disparition de l'objet lui-même. De ce point de vue, le statut du zeppelin est semblable à celui des dinosaures, celui d'une espèce éteinte qui a disparu de l'horizon de notre société contemporaine, à l'exception des dirigeables à vocation publicitaire ou touristique. L'autre perte est l'effondrement de l'utopie dont le zeppelin était porteur et qui associait le progrès technologique au devenir pacifique et solidaire de l'Europe. Cette utopie a été détruite par la contre-utopie du nazisme qui s'est elle-même abîmée dans une catastrophe de feu et de flammes. En raison de ce double effondrement, le zeppelin semble coupé de tout devenir historique, ce qui favorise sa réapparition dans l'inactualité de mondes parallèles marqués du sceau du rétrofuturisme.

En termes de culture visuelle, la question est dorénavant de savoir dans quelle mesure l'imagerie que nous avons précédemment dégagée - l'imagerie, c'est-à-dire ici l'association d'une image avec un certain type de discours, d'émotion et d'acteur - se trouve également gagnée par un sentiment de déperdition. Autrement dit, il s'agit d'examiner comment se reconfigure l'affect du sublime lorsque son objet a disparu, comment aussi se reformule le discours utopique quand cette utopie a été frappée d'impuissance. Parallèlement, on peut se demander si le zeppelin peut donner lieu à de nouvelles utopies et partant à de nouvelles

16 Voir par exemple le neuvième volume (2004) de la série Capricorne dont la couverture s'orne d'un dirigeable. 
images ou s'il est condamné à être recyclé indéfiniment dans un même type d'univers visuel.

\subsection{Le zeppelin en miroir déformant du passé}

Pour répondre à ces questions, la première tentation serait de placer l'ensemble des réactualisations du zeppelin sous le registre d'une contemplation mélancolique des utopies passées et d'une méditation sur les ruines du progrès. Fréquemment définie comme une tristesse sans objet, la mélancolie entretient un rapport privilégié avec le discours utopique dans la mesure où l'utopie non réalisée développe chez le penseur un sentiment de perte portant sur ce qui aurait pu être et n'a pas été. Dans The Future of Nostalgia (2001), Svetlana Boym définit la personne mélancolique comme « un rêveur utopiste qui avait de plus hauts espoirs pour l'humanité $\gg{ }^{17}$ (p. 5). L'affect du sublime est bien présent dans un tel processus, puisque l'on peut soutenir avec Julia Kristeva (2001) que sublime et mélancolie se relancent mutuellement pour accroître le sentiment de perte lié à un deuil impossible ${ }^{18}$.

En bande dessinée, le cycle des Cités obscures de Schuiten et Peeters peut tout à fait s'interpréter sous ce jour mélancolique. Par exemple, dans La route d'Armilia (1988), un enfant - qui est aussi le narrateur du récit - embarque dans un zeppelin pour un voyage au-dessus d'un continent ressemblant étrangement à l'Europe. L'imaginaire du zeppelin développé à partir du texte de Zweig est actualisé, mais à travers le filtre de la désolation, puisque, mis à part quelques figures isolées, aucune foule n'acclame le passage du dirigeable. Le voyage jusqu'à la cité arctique d'Armilia s'avère au final le rêve chimérique de l'enfant connecté à une machine futuriste. Pour dépeindre ce voyage, les

17 Ma traduction de l'anglais : « a utopian dreamer who had higher hopes for humanity ».

18 De son côté, Paul Ricœur (2000) associe la mélancolie à la tristesse de la mémoire méditative, une tristesse qui peut éventuellement se sublimer en gaieté. La sublimation, qui n'est pas tout à fait le sublime, fournit alors le « secret du retournement de la complaisance à la tristesse en tristesse sublimée - en gaieté » (p. 94). 
auteurs ne se contentent pas de s'approprier l'esthétique Art déco associée à l'époque des zeppelins. Ils transposent aussi le sublime monumental du dirigeable par un travail graphique approprié. Pour amplifier le caractère hors norme du zeppelin, les auteurs jouent sur de doubles pages ou font en sorte que la machine ne puisse entièrement tenir dans le cadre. Un sentiment de mélancolie méditative émane alors de l'album, suscité en grande partie par des images où le zeppelin paraît à l'arrêt au-dessus d'un continent qui se trouve comme menacé d'engourdissement et de gel.

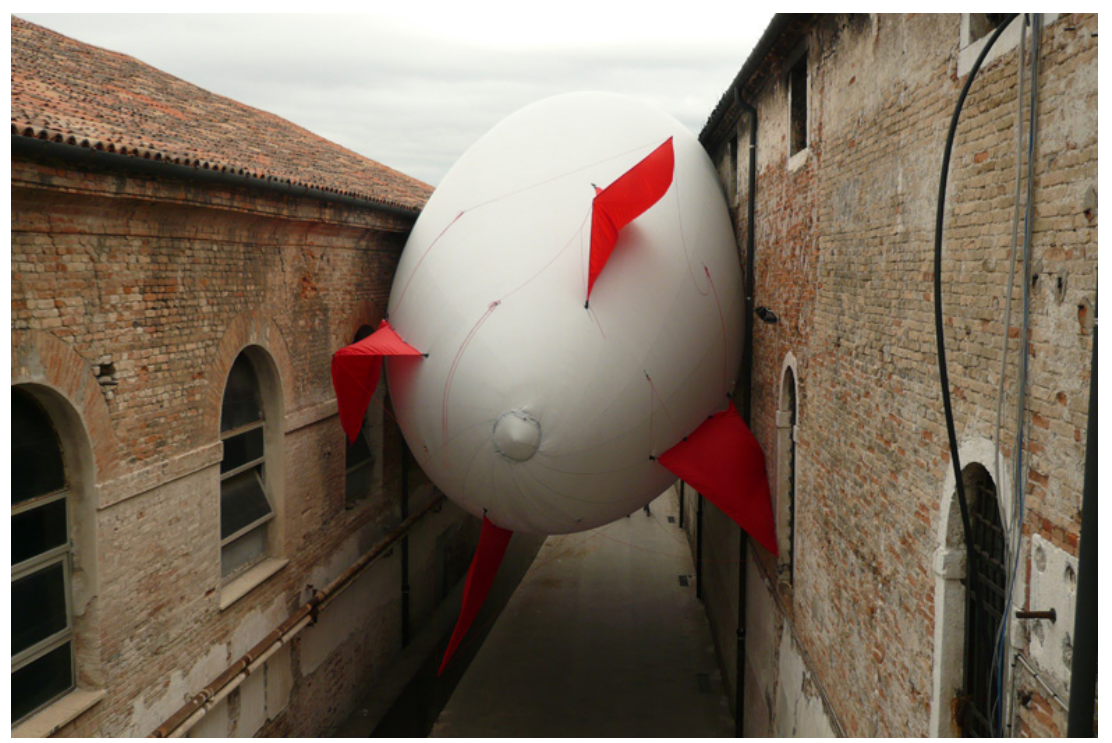

Figure 4. Sciame di Dirigibili par Héctor Zamora (2009)

Toutefois, la tonalité mélancolique n'est pas la seule à survenir lorsque le zeppelin rejoue les utopies passées et qu'il mobilise l'imaginaire du voyage relié aux dirigeables. Ainsi, Héctor Zamora, un artiste mexicain, propose pour la Biennale de Venise en 2009 une installation baptisée Sciame di Dirigibili (Zeppelin Swarm) ${ }^{19}$. L'artiste reprend à son compte le souvenir des grandes foires aéronautiques d'autrefois et imagine qu'une

19 Un portfolio de l'artiste, comprenant des images de l'exposition Sciame di Dirigibili, est disponible à l'adresse suivante : www.lsd.com.mx/portfolio_Hector_Zamora.pdf (consulté le 10 mai 2017). 
manifestation autour des zeppelins s'est tenue à Venise. Des affiches rétro, des cartes postales témoignent d'un événement qui en réalité n'a jamais eu lieu. La seule trace effective de cette manifestation est un zeppelin gonflable coincé dans une ruelle qui apparaît comme le résidu de l'essaim disparu (voir figure 4).

À travers cette installation, l'artiste s'approprie l'univers visuel des zeppelins, mais dans une perspective ironique combinant à la fois adhésion et distanciation. Les images recréent la grandeur des foires aéronautiques d'autrefois, mais le zeppelin piégé dans la ruelle présente une tonalité burlesque qui contraste avec la majesté supposée de la machine. Le zeppelin est figuré comme un objet qu'on ne sait plus désengager de l'espace urbain, une utopie encombrante dont on ne parvient pas à se débarrasser. Comme l'a bien montré Fredric Jameson, l'ironie renvoie alors à cette forme du modernisme tardif « qui permet d'être utopiste et anti-utopiste, et de planer, pendant un ultime instant, dans cet espace suspendu où nous sommes à la fois les deux et ni l'un ni l'autre, dans cet espace où les dés ne sont pas encore jetés (et ne le seront jamais) » (2007, p. 308).

Sous un registre d'images plus proche du paradigme de la catastrophe que de celui du voyage, le zeppelin est également réactivé dans des univers rétro-futuristes non pas tant sur le mode de la mélancolie que sur celui de l'angoisse, comme signe d'une catastrophe qui aurait pu survenir si les temps avaient pris une autre tournure ${ }^{20}$. L'utopie devient alors uchronie ${ }^{21}$. Certains romans comme Le faiseur d'Histoire (2011) de Stephen Fry montrent par exemple la victoire du Troisième Reich et l'occupation de Londres par les nazis, ce qui donne lieu à certaines images (de couverture notamment) représentant des zeppelins victorieux ornés d'une croix gammée flotter au-dessus de la capitale britannique. Le sentiment suscité ici est très proche de l'inquiétante étrangeté décrite par Freud (1985), à savoir le

20 La dimension uchronique apparaît avec évidence dans la série Fringe où des dirigeables croisent dans le ciel d'un New York alternatif où les Twin Towers du World Trade Center ne se seraient pas effondrées.

21 Pour un éclairage récent sur l'uchronie et les futurs non advenus, voir l'ouvrage de Quentin Deluermoz et Pierre Singaravélou (2016). 
retour non intentionnel du même, comme si une part sombre de notre histoire que l'on croyait disparue ressurgissait brusquement dans un présent parallèle au nôtre.

Parfois, ce mode catastrophique se décline de façon plus ludique. Dans l'une des séquences du film Sucker Punch (Snyder, 2011), les héroïnes se trouvent projetées dans une tranchée de la Grande Guerre où les soldats allemands ont été transformés en zombies mécanisés. Elles finissent par affronter un dirigeable qui s'écrase dans une scène rejouant le crash du Hindenburg. Ici aussi, le sublime est bien présent : on pourrait même parler d'un sublime numérique, car la séquence est conçue comme une attraction (Strauven, 2006 ; Gaudreault, 2008), un pur moment de déchaînement visuel rendu possible par la technologie numérique. Le zeppelin vaut surtout pour la destruction spectaculaire dont il est l'objet, dans un récit qui au demeurant n'est pas spécialement focalisé sur la période de la Première Guerre.

Ces premières analyses montrent comment les séries du voyage et de la catastrophe continuent à être mobilisées autour de la figuration du zeppelin à l'époque contemporaine en faisant ressortir une gamme d'émotions qui accentuent la sensation de déperdition. La foule cède la place à des espaces vides et désolés, comme si on prenait acte d'un échec collectif ; la composante urbaine reste dominante, mais placée sous le signe d'une revisitation des architectures du passé. Quant au sentiment du sublime, il se maintient bel et bien, mais il n'est plus lié aux prodiges technologiques de la machine, mais davantage au pouvoir sidérant de la représentation en elle-même qui maintient, notamment grâce au trucage numérique, l'impact visuel du dirigeable. Tout se passe donc comme si les représentations actuelles du zeppelin étaient dans une relation de miroir vis-àvis du passé, un miroir déformant certes, mais qui privilégie une stratégie du reflet et de la distorsion, plutôt que du dépassement et de l'ouverture. 


\subsection{Le dirigeable, véhicule des utopies de demain}

Reste posée la question de savoir dans quelle mesure l'image du zeppelin peut se trouver associée à de nouvelles utopies permettant d'ouvrir le champ des possibles. Selon nous, cette question suppose de considérer que l'ouverture d'une dimension utopique supplémentaire passe par le renouvellement de l'imagerie préexistante, ce qui oblige alors les représentations du zeppelin à adopter d'autres modalités de configuration pour s'adapter à des discours qui tendent à se projeter davantage vers l'avenir que le passé.

Un autre champ remarquable d'apparition du zeppelin est à l'œuvre dans les plans des architectes et des urbanistes qui conçoivent le monde de demain. À l'heure du réchauffement climatique, le zeppelin apparaît comme un mode de transport alternatif à l'aviation en développant une conception du voyage moins axée sur la vitesse. Dans un article intitulé « Les dirigeables : alternative aux avions de lignes ? » (2008), l'écologiste George Monbiot soulignait le coût environnemental relativement faible de tels engins et remarquait qu'un " voyage en dirigeable serait plutôt comme un voyage en bateau de croisière, mais avec une vitesse deux fois plus grande et l'utilisation d'une fraction seulement de carburant. »

Sur un plan discursif, il est intéressant d'observer que le dirigeable se trouve investi d'une valeur écologique qu'il ne possédait pas auparavant et qui le dote d'une potentialité future. Ce réinvestissement sémantique s'accompagne fréquemment d'un changement lexical : lorsqu'il s'agit d'évoquer des utopies pour le monde de demain, le terme 'dirigeable' (ou 'airship') est préféré au terme 'zeppelin', sans doute trop lourdement connoté d'un point de vue historique. Cependant, ce changement de nom n'implique pas une révocation de l'imagerie associée au zeppelin, mais davantage une évolution qui reconfigure certains traits des représentations préexistantes. Les images associées aux nouveaux dirigeables ne peuvent éviter de réactiver dans la mémoire du spectateur des images plus anciennes, liées à la grande époque des zeppelins. En même temps, ces nouvelles images se détachent des 
précédentes en faisant surgir d'autres caractéristiques visuelles plus en accord avec les préoccupations contemporaines.

Ceci se vérifie par exemple dans le projet d'Adam Holloway, jeune urbaniste, qui a proposé en 2008 un Anemorphic Airship Docks pour Londres. L'idée consiste à construire un aéroport pour dirigeables sous la forme d'une structure en spirale qui pivote légèrement en fonction des vents de la City. Dans la série de planches et de dessins préparatoires qui composent le projet $^{22}$, on observe que les images réactivent bien la composante urbaine et architecturale associée à l'image du dirigeable, mais en présentant de nouveaux immeubles dont les formes organiques évoquent le milieu naturel et non l'univers d'acier et de béton qui apparaissait dans les années d'avant-guerre (voir figure 5). Plus subtilement, les images montrent aussi des figures humaines intégrées dans l'installation aéroportuaire. Contrairement à ce qui passait dans l'album de Schuiten et Peeters, la foule n'est pas évacuée de l'image, mais se trouve convertie en une multitude d'usagers s'apprêtant à utiliser les nouvelles infrastructures.

Il convient d'ailleurs de s'arrêter plus longuement sur la question de l'infrastructure. Dans le projet d'Adam Holloway, le dirigeable n'est qu'une partie d'un dispositif plus global visant à associer transport écologique et nouvelle zone aéroportuaire. On trouve une même logique dans le projet Airbia (2009) conçu par Alexandros Tsolakis et Irene Shamma ${ }^{23}$. Ici, le projet est de faciliter le déplacement des habitants des banlieues vers les centres urbains grâce à une flotte de dirigeables volant à haute altitude. Une telle ambition est illustrée par des images de synthèse qui montrent par exemple un dirigeable faisant route vers le centreville ou atterrissant dans une banlieue résidentielle.

22 Pour un aperçu du projet d'Adam Holloway, voir www.presidentsmedals.com/ Project_Details.aspx?id=2265 (consulté le 10 mai 2017).

23 Pour un aperçu du projet Airbia, voir http://inhabitat.com/airbia-suburban-airshipstake-flight/ (consulté le 10 mai 2017). 


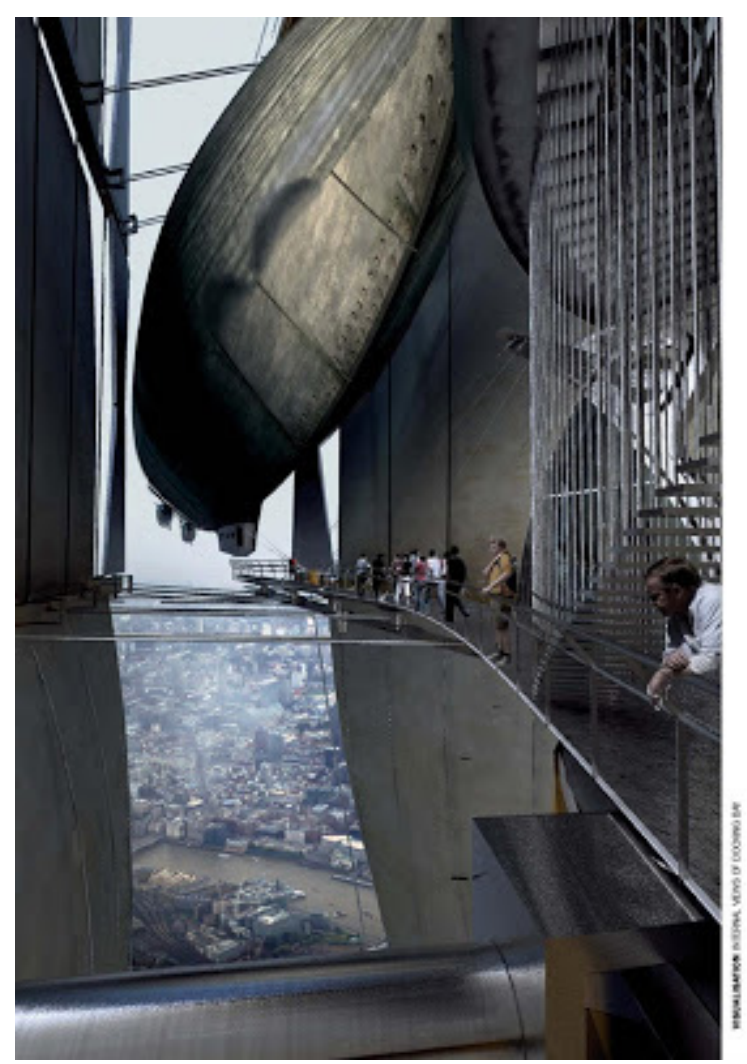

Figure 5. Anemorphic Airship Docks d'Adam Holloway (2008)

Dans ces images (et les projets qui les sous-tendent), l'accent utopique se déplace vers les infrastructures. Il ne s'agit pas tellement de célébrer les prouesses technologiques du dirigeable, mais la façon dont celui-ci peut s'intégrer à de nouveaux espaces urbains respectueux de l'environnement. Pour certains penseurs contemporains (dont l'architecte et sociologue Yona Friedman ${ }^{24}$ ),

24 Selon Friedman, il est certain que le renouveau de l'humanité ne passera pas par un nouvel état mondial qui permettrait d'apaiser les différences entre les nations, une utopie qui rappelle d'une certaine manière l'idéal de solidarité européenne évoqué par Zweig. Par contre, écrit Friedman, il est tout à fait possible de mettre en place un organisme de gérance mondiale, «à condition que la compétence de cette organisation ne dépasse pas la maintenance des voies d'accès reliant les territoires entre eux (et servant à l'échange des moyens de survie) ».

Friedman cité dans Jameson (2007, pp. 370-371). 
l'élan utopique n'est plus à chercher du côté de l'invention de nouvelles formes d'organisation sociale; il est davantage associé au renouvellement des infrastructures et des voies d'aménagement donnant à tous l'occasion de coexister harmonieusement. Comme le résume Fredric Jameson, c'est bien « l'infrastructure - autrement dit, la planète elle-même et sa matérialité ), qui permettra de combiner les diverses utopies séparées » (2007, p. 370). Dans cette perspective, l'accent mis sur l'infrastructure dans les projets liés aux dirigeables rejoint pleinement ce qui caractérise aujourd'hui les nouvelles utopies.

Pour revenir aux images, il faut noter que ces visions prospectives sont soutenues par un régime visuel différent de celui qui animait les productions précédentes. En effet, les projets urbanistiques plaçant le dirigeable en leur centre sont illustrés par des plans, des dessins vectoriels, des vues d'architecte, des images de synthèse qui documentent paradoxalement une réalité à venir. Ces images s'annoncent comme les premières traces d'une ville qui existera peut-être dans le futur. Le mode d'être de ces images s'apparente donc à une virtualité en attente de sa réalisation concrète. Contrairement à la bande dessinée La route d'Armilia ou à l'installation d'Héctor Zamora, ces images ne font pas mine d'entériner un événement qui aurait déjà eu lieu. En tant qu'images-projets, elles comportent une dimension performative qui renvoie précisément à l'acte de créer et d'imaginer de nouvelles utopies.

Dans Le futur n'existe pas. Rétrotypes (2014), l'artiste contemporain Alain Bublex et le philosophe Elie During proposaient de considérer certaines œuvres visuelles non comme des prototypes, mais comme des rétrotypes en arguant que le rétrotype permet d'exhiber à l'état potentiel une idée du passé qui continue d'agir comme projet pour le monde futur. Si l'on considère cette proposition selon le paradigme de l'imagerie évoquée par Pascal Ory, nous pouvons alors faire valoir que le renouveau de l'imagerie du dirigeable passe par le retour à un état antérieur de l'image, comme s'il fallait, pour relancer le zeppelin sur de nouvelles voies, revenir à un régime visuel permettant de reconsidérer sous un jour nouveau les potentialités de la 
machine. Ces potentialités étaient présentes dès le départ, lorsque le zeppelin n'était qu'un plan dessiné sur une feuille de papier, mais elles avaient été comme ensevelies sous les représentations successives du dirigeable. Bizarrement donc, s'ouvrir au devenir suppose de revenir en arrière, à un temps antérieur qui précède et conditionne les paradigmes du voyage et de la catastrophe.

Là est peut-être la torsion la plus forte du rétro-futurisme qui consiste à retrouver dans l'image contemporaine tendue vers le futur le même état de prospective qui avait été celui des inventeurs de l'appareil. Le prototype devient alors rétrotype et vice-versa, dans un entre-temps qui s'exprime d'abord sous le mode du virtuel et du devenir.

\section{Conclusion : le réseau imaginaire de l'objet}

En procédant à un montage d'images au sens entendu par Georges Didi-Huberman et en se focalisant sur les variations et reprises d'un même motif à travers le temps, notre analyse s'est attachée à comprendre comment se maintient, mais aussi comment se renouvelle l'imagerie d'un objet. Tout au long de l'article, il est apparu avec netteté que l'imagerie du zeppelin maintient un rapport relativement stable avec certaines composantes formant ce que l'on pourrait appeler le réseau imaginaire de l'objet, un réseau qui se trouve constitué, dans le cas du zeppelin, par un certain type d'acteur, un rapport spécifique à l'environnement urbain et l'association à une dimension utopique. Ces différents éléments peuvent certes se reconfigurer, mais ils donnent à l'image du zeppelin dans la culture contemporaine une assise relativement stable qui le positionne dans la continuité des représentations passées.

En même temps, l'imagerie est aussi animée par un processus d'invention et de créativité qui permet au zeppelin de ne pas être totalement figé dans le cliché visuel ou piégé dans le cycle de la répétition. Cette part d'invention peut par exemple se traduire par la modification de l'un des éléments constitutifs du réseau imaginaire de l'objet (comme la disparition de la foule chez Schuiten et Peeters). Cependant, il nous semble que l'imagerie se 
trouve profondément renouvelée lorsqu'elle parvient à développer des potentialités figuratives qui se connectent à de nouveaux discours œuvrant dans l'espace social. Nous rencontrons alors au niveau des images le concept de rétroaction que Paul Ricœur associe à l'imaginaire social. Pour Ricœur, il s'agit de délivrer des possibles qui avaient été empêchés, mais qui se trouvaient présents depuis le départ : «Pour être créateur, il faut pouvoir faire mémoire de ce que nous avons abandonné afin de l'inclure dans ce que nous avons trouvé " (Castoriadis \& Ricœur, 2016, p. 64). C'est précisément ce qui se produit avec les images du zeppelin soutenant les nouveaux projets urbanistiques. Les composantes du réseau imaginaire de l'objet sont reconsidérées d'un œil neuf, car elles s'insèrent dans un nouveau contexte énonciatif, celui de l'écologie, qui amène à reprendre l'image 'à zéro', dans un état antérieur à celui de ses incarnations historiques successives.

Dans le réseau imaginaire, les émotions jouent un rôle central dans le sens où elles participent aussi à donner une certaine continuité à l'imagerie de l'objet ou plutôt à sa perception. Dans le cas du zeppelin, c'est assurément le sublime qui joue ce rôle d'affect récurrent. Toutefois, il faut de nouveau insister sur le fait que le jeu des émotions n'est jamais totalement figé. Si l'on s'en tient à la culture contemporaine, on s'aperçoit que les modalités affectives du zeppelin découlent certes du sublime suscité par la vision d'un objet hors norme, mais que ce sublime peut évoluer entre angoisse et mélancolie en fonction du contexte de représentation. C'est peut-être à ce niveau que les perspectives d'exploration future sont les plus intéressantes. Si des historiens comme Alain Corbin (2016) travaillent déjà sur l'histoire des émotions en privilégiant la construction sociale d'une émotion particulière comme la peur ou le ravissement, l'approche développée ici permet de cartographier la gamme d'affects que peut susciter la représentation d'un même objet, en tenant compte de son positionnement historique. Une telle approche demanderait certes à être étayée par d'autres cas pratiques, mais il nous semble que l'analyse de l'image du zeppelin a déjà permis d'ouvrir la voie à une approche modulaire des émotions intégrant l'évolution de l'objet et celle de ses représentations. 


\section{Références}

Aujaleu, E. (1997). La sensibilité esthétique et le jugement de goût. Dans Tréma, n 11 , pp. 33-52.

Barillier, E., \& Colson, R. (2014). Tout le steampunk! Montélimar : Les Moutons électriques.

Belting, H. (2004) [2001]. Pour une anthropologie des images. Paris : Gallimard.

Boym, S. (2001). The Future of Nostalgia. New York : Basic Books.

Bublex, A., \& During, E. (2014). Le futur n'existe pas. Rétrotypes. Paris : Éditions B42.

Castoriadis, C., \& Ricœur, P. (2016). Dialogue sur l'histoire et l'imaginaire social. Paris : Éditions de l'École des hautes études en sciences sociales.

Corbin, A., Courtine, J.-J., \& Vigarello, G. (Éd.). (2016). Histoire des émotions. Des Lumières à la fin du XIXe siècle. Vol. 2. Paris : Seuil.

Delrue, F. (2015). Le futur n'existe pas. Recension de l'ouvrage d'A. Bublex \& E. During. Le Futur n'existe pas : rétrotypes. Dans Implications philosophiques. Espace de recherche et de diffusion. Disponible à : http://www.implications-philosophiques. org/recensions/le-futur-nexiste-pas/.

Deluermoz, Q., \& Singaravélou, P. (2016). Pour une histoire des possibles. Analyses contrefactuelles et futurs non advenus. Paris : Seuil.

Dessy, Cl., \& Stiénon, V. (Éd.) (2015). (Bé)vues du futur. Les imaginaires visuels de la dystopie (1840-1940). Villeneuve-d'Ascq : Presses Universitaires du Septentrion.

De Syon, G. (2002). Zeppelin! Germany and the Airship, 1900-1939. Baltimore \& London : Johns Hopkins University Press.

Didi-Huberman, G. (2003). Images malgré tout. Paris : Éditions de Minuit.

Freud, S. (1985) [1919]. L'inquiétante étrangeté. Dans S. Freud, L'inquiétante étrangeté et autres essais. (pp. 209-263). Paris : Gallimard.

Fry, S. (2001) [1996]. Le faiseur d'Histoire. Paris : Folio SF.

Gaudreault, A. (2008). Cinéma et attraction. Pour une nouvelle histoire du cinématographe. Paris : CNRS.

Hiam, C. M. (2014). Dirigible Dreams. The Age of the Airship. Lebanon : ForeEdgeUniversity Press of New England.

Jameson, Fr. (2007) [2005]. Archéologies du futur. Le désir nommé utopie. Paris : Max Milo Éditions.

Kazenwadel-Drews, B. (2006). Zeppeline Erobern die Welt. Bielefeld : Delius Klasing Verlag.

Kristeva, J. (2001). La traversée de la mélancolie. Figures de la psychanalyse, vol. 1, $\mathrm{n}^{\circ} 4,19-24$.

Leblanc, P. (1994). L'imaginaire social. Note sur un concept flou. Dans Cahiers internationaux de Sociologie, vol. XCVII, pp. 415-434.

Michel, J. (2016). Préface. Dans C. Castoriadis \& P. Ricœur, Dialogue sur l'histoire et l'imaginaire social. (pp. 7-34). Paris : Éditions de l'École des hautes études en sciences sociales.

Monbiot, G. (2008) [2008]. Les dirigeables : alternative aux avions de lignes ?. Dans DDmagazine. Le développement durable en pratique. Disponible à http://www. ddmagazine.com/317-Les-dirigeables-alternative-aux-avions-de-lignes.html.

Nye, D. E. (1994). American Technological Sublime. Cambridge/London : MIT Press. 
Ory, P. (2015) [2004]. L'histoire culturelle. Paris : PUF.

Ricœur, P. (2000). La mémoire, l'histoire, l'oubli. Paris : Seuil.

Schuiten, Fr., \& Peeters, B. (1988). La route d'Armilia et autres légendes du monde obscur. Bruxelles : Casterman.

Strauven, W. (Éd.) (2006). The Cinema of Attractions Reloaded. Amsterdam : Amsterdam University Press.

Traverso, E. (2016). Mélancolie de gauche. La force d'une tradition cachée (XIX ${ }^{e}-X X I^{e}$ siècle). Paris : La Découverte.

Zweig, S. (1993) [1944]. Le Monde d'hier. Souvenirs d'un Européen. Paris : Le Livre de Poche.

\section{(9) $\Theta \Theta$}

Publié sous la licence Creative Commons

«Attribution - Pas d'Utilisation Commerciale - Pas de Modification 4.0 International» (CC BY-NC-ND) 